Check for updates

Cite this: RSC Adv., 2019, 9, 29217

Received 8th May 2019

Accepted 9th September 2019

DOI: $10.1039 / c 9 r a 03447 a$

rsc.li/rsc-advances

\section{Erythrocyte membrane affinity chromatography, solid-phase extraction and UPLC-QTOF-MS/MS to screen active ingredients of Buyang Huanwu decoction $\dagger$}

\author{
Aiming Yu, (D) Huazhu Zheng, Xiangli Yan, Shengxin Wang, Xiao Shen, \\ Haozhen Zheng, Yingjiao Meng and Lisheng Wang (D)*
}

Buyang Huanwu decoction (BHD) is a well-known traditional Chinese medicine that has long been used to treat ischemic brain damage which is associated with hemorheology. To screen active ingredients in BHD responsible for reducing blood viscosity by reducing red blood cell (RBC) lesions to treat ischemic stroke, a method involving RBC membrane binding and solid-phase extraction (SPE) was developed in this study. The components of BHD interacting with RBC were analyzed by mass spectrometry and four compounds, calycosin, paeoniflorin, 6-hydroxy behenol-3,6-di-O-glucoside and calycosin-7-O- $\beta$-D-glucoside, showed binding affinity to RBCs. An erythrocyte activity assay revealed that the identified ingredients promoted the activities of $\mathrm{Na}^{+}-\mathrm{K}^{+}$-ATPase, sialic acid and superoxide dismutase and reduced the content of cholesterol on the RBC membrane, suggesting a mechanism underlying their anti-erythrocyte aggregation activity. Based on these results, the RBC membrane binding assay combined with SPE and mass spectrometry is a novel and effective approach for screening potentially anti-erythrocyte lesion constituents in traditional Chinese medicines.
\end{abstract}

\section{Introduction}

Buyang Huanwu decoction (BHD) is a reputable traditional Chinese medicine that has long been used to treat ischemic brain damage and repair brain post-injury. ${ }^{\mathbf{1 , 2}}$ Many researchers are interested in the compositions of BHD but whether these ingredients are bioactive and how they improve stroke rehabilitation are not further verified. Typical compounds in BHD such as astragaloside IV, calycosin-7- $O-\beta$-D-glucoside, ferulic acid, etc., were demonstrated to be responsible for the effects on preventing platelet aggregation thereby promoting stroke recovery. Antiplatelet therapy is the main clinical strategy used to treat stroke patients but the effect is unsatisfactory, ${ }^{3-5}$ and thus more effective compounds in BHD with different targets are needed to be systematically elucidated, which would help interpret the therapeutic compounds of BHD for treating strokes.

Ischemic stroke (IS) is a type of brain disease that develops rapidly and results in a high rate of disability, making it the second most common cause of death worldwide. ${ }^{6}$ The pathogenesis of IS is complex, and previous studies have mainly

School of Pharmaceutical Sciences, Guangzhou University of Chinese Medicine, No. 232, Waihuan East Road, Panyu District, Guangzhou 510006, China. E-mail: wlis68@126.com

$\dagger$ Electronic supplementary information (ESI) available. See DOI: 10.1039/c9ra03447a focused on the relationship between changes in hematological parameters and development of ischemic stroke. ${ }^{7}$ Hemorheological abnormalities, such as platelet aggregation, changes in global viscosity, hematocrit, and erythrocyte aggregation, are closely associated with the pathogenesis of ischemic cerebrovascular disease. Despite recent advances, drugs commonly used to treat IS by dissolving fibrin in thrombus is facing great challenges due to drug resistance. ${ }^{8}$ It was found that red blood cell (RBC)-rich thrombus has increased viscosity and deformability and reduced elasticity and stiffness. ${ }^{9}$ A previous study revealed an increase in erythrocyte agglutination in patients with stroke, indicating that microcirculation is affected by enhanced erythrocyte agglutination, reduced RBC deformability, and subsequent circulation disorder. Moreover, erythrocyte agglutination activates the exogenous coagulation system, follow by increasing platelet adhesion and thrombus formation, which eventually lead to microvascular perfusion disorders. ${ }^{10}$ Decreased $\mathrm{Na}^{+}-\mathrm{K}^{+}$-ATPase activity and sialic acid (SA) and increased cholesterol (CHO) contents that affect the deformability of RBCs, are responsible for erythrocyte aggregation. ${ }^{11-13}$ Because the most commonly used thrombolytic therapy, which targets platelets, has shown unsatisfactory effects, studies of changes in RBCs are needed to provide a new strategy for treating IS.

The capacity of drugs to bind to specific targets on the cell membranes is key for their curative effects in vivo. ${ }^{\mathbf{4}}$ For 
instance, $\mathrm{Na}^{+}-\mathrm{K}^{+}$-ATPase is an important functional receptor on the cell membrane that most cardiac glycosides specifically binding to; ${ }^{15}$ beta 2 adrenergic receptor on erythrocyte membrane is a specific target for Terbutaline in the treatment of lung diseases. ${ }^{16,17}$ These finding suggests that cell membranes can be used as a stationary phase to screen for active pharmaceutical ingredients in medicinal chemistry research. Many researchers obtained a cell membrane stationary phase by packing the silica gel and cell membrane into the CMC column, but this system is unstable and the encapsulating, fixing material and gel matrix must be adapted to the type of material to be analysed..$^{18}$ Directly incubating the cell membrane with the herbal extract without complicated procedures of preparing bio-chromatographic columns can meet the requirements of pressure and ionic strength for cell membrane. ${ }^{\mathbf{1 9 , 2 0}}$ Based on this, RBC affinity chromatography to isolate active ingredients that relieve blood circulation after IS is an efficient method for studying the interactions between numerous compounds and proteins on the membrane. ${ }^{21-23}$ Specific components of BHD that lower blood viscosity can be separated from the other compounds on the basis of binding to respective targets on RBCs.

The purpose of this study was to isolate and identify bioactive components in BHD that reduce blood viscosity and improve IS. Effective pre-treatment methods for purification and enrichment, such as elution procedures for solid-phase extraction (SPE) columns, were developed and erythrocyte membranes were extracted to screen potentially active components in BHD. A total of four affinity components combined with erythrocyte membranes were isolated and found to be bioactive. These findings provide insight into the bioactive components of BHD and contribute to an effective method of screening effective drugs to treat disease caused by erythrocyte lesion.

\section{Experimental}

\section{Materials and reagents}

Radix Astragali (root of Astragalus membranaceus), Radix Paeoniae Rubra (root of Paeonia lactiflora pall), Ligusticum wallichii (root and stem of Ligusticum chuanxiong Hort.), Radix Angelica sinensis (root of Angelica sinensis), Lumbricus, Semen Persicae (dry seeds of Prunus persica), and Flos Carthami (flower of Carthamus tinctorius L.) were purchased from Guangzhou Zhixin Chinese Herbal Medicine Co., Ltd. (Guangzhou, China) and identified as authentic by Professor Danyan Zhang of Guangzhou University of Chinese Medicine. D101 macroporous resin was purchased from Shanghai Hualing Resin Co., Ltd. (Guangzhou, China). Phosphate-buffered saline (PBS) was from Gibco (Grand Island, NY, USA) and fresh blood from healthy pigs was provided by Guangzhou Panyu Food Group Co., Ltd. (Guangzhou, China). Formic acid, acetonitrile, and methanol of chromatographic grade were obtained from Thermo Fisher Scientific (Waltham, MA, USA) and Merck (Darmstadt, Germany), respectively. Other reagents and chemicals such as anhydrous ethanol were of analytical grade. The SPE columns were from Phenomenex, Inc. (Torrance, CA, USA).
Calycosin, calycosin-7-O- $\beta$-D-glucoside, paeoniflorin, and 6hydroxy kaempferol-3,6-di-O-glucoside (over 98\% in purity) were purchased from Chengdu Pfeide (Sichuan, China).

\section{Chromatographic conditions and analytical system}

High-resolution mass spectrometry (MS) is widely used to identify unknown compounds because of its high sensitivity compared to low-resolution MS. In this study, quadrupole timeof-flight (QTOF)-MS was used to identify the components in the dissociation solution of BHD combined with erythrocyte membranes.

Ultra-performance liquid chromatography (UPLC) was carried out with a binary gradient pump, auto plate-sampler, and DAD detector (Shimadzu, Kyoto, Japan). The analysis was performed on a Kinetex C18 column $(2.1 \times 50 \mathrm{~mm}, 2.6 \mu \mathrm{m})$, with gradient elution with the mobile phase comprising of (A) methanol and (B) water (containing $0.1 \%$ formic acid) at a flow rate of $0.3 \mathrm{~mL} \mathrm{~min}{ }^{-1}$. The injection volume was $3 \mu \mathrm{L}$ and the detection temperature was $35{ }^{\circ} \mathrm{C}$. The elution parameters were as follows: $10-40 \%$ A at $0-5 \mathrm{~min}, 40-60 \%$ A at 5-10 $\mathrm{min}, 60-40 \%$ $\mathrm{A}$ at $10-15 \mathrm{~min}, 40-10 \% \mathrm{~A}$ at $15-20 \mathrm{~min}$.

QTOF-MS analysis was performed with an AB SCIEX Triple TOF 5600+ system (AB SCIEX, Inc., Framingham, MA, USA) equipped with an electrospray ionization source in both positive and negative modes. The conditions were as follows: ion source gas $1\left(\mathrm{~N}_{2}\right)$ pressure, $378.95 \mathrm{kPa}$; ion source gas 2, 378.95 $\mathrm{kPa}$; curtain gas, $241.15 \mathrm{kPa}$; drying gas temperature, $600{ }^{\circ} \mathrm{C}$; ion spray voltage floating, $5.5 \mathrm{kV}$; declustering potential, $100 \mathrm{~V}$; collision energy, $10 \mathrm{eV}$. The scan range was set to 100-1000. Data acquisition and analysis were conducted with Analyst TF1.7 software (AB SCIEX) and PeakView software.

\section{Sample preparation}

BHD consists of Radix Astragali, Radix Paeoniae Rubra, Ligusticum wallichii, Radix Angelica sinensis, Lumbricus, Semen Persicae, and Flos Carthami at a $60: 6: 9: 9: 9: 9: 9$ ratio. BHD was extracted from the herbs according to our previous study. ${ }^{23}$ Briefly, the herbs were immersed in distilled water and then boiled twice, the first time with the equivalent of 8 times the amount of water and second time with 6 times the amount of water. Next, the filtrate was concentrated and treated with $85 \%$ ethanol to remove impurities. The solution was concentrated and then purified with D101 macroporous resin. Next, the samples were washed with water until the results of the Molisch reaction were negative, and $40 \%$ and $70 \%$ ethanol eluents were collected. Ethanol was removed, and the samples were analysed after dilution to $0.555 \mathrm{~g} \mathrm{~mL}^{-1}$ and filtering through a $0.22 \mu \mathrm{m}$ membrane. According to our previous study, ${ }^{23}$ high-performance liquid chromatographic fingerprint (HPLC) was conducted to control the quality of the extracted BHD. Additionally, a sample solution of each herbal medicine was extracted as described for BHD extraction.

\section{Preparation of erythrocyte membrane}

Erythrocyte membranes were extracted by using hyperosmotic solution to hemolyze erythrocyte with modification of 
Agnihotri's method. ${ }^{24}$ Briefly, approximately $2500 \mathrm{~mL}$ of fresh pig blood was collected in PBS (pH 7.4) containing heparin and stored at $4{ }^{\circ} \mathrm{C}$ overnight. The blood was centrifuged to obtain RBCs on the next day. The pellet was washed three times with ice-cold PBS ( $\mathrm{pH} 7.4$ ), followed by hemolysis in ice-cold $0.25 \times$ PBS (pH 7.4) for $1 \mathrm{~h}$. The supernatant was discarded after centrifugation at $3819 \mathrm{rpm}$ for $5 \mathrm{~min}$, and the RBC pellet was washed three times with PBS to obtain erythrocyte membranes for subsequent experiments.

\section{Erythrocyte membrane binding assay}

RBC binding assay was carried out according to a method by Liao et al. with modification. ${ }^{19}$ Briefly, the RBC membrane was resuspended into suspension in $\mathrm{PBS}(\mathrm{pH} 7.4)$, and $5 \mathrm{~mL}$ of the RBC membrane suspension was added to 4 or $5 \mathrm{~mL}$ of BHD $\left(1.1 \mathrm{~g} \mathrm{~mL}^{-1}\right)$ sample solution and incubated at $37^{\circ} \mathrm{C}$ for $45 \mathrm{~min}$ in a water bath to fully bind the active ingredients in the erythrocyte membrane. The control group was incubated with an equal amount of PBS ( $\mathrm{pH}$ 7.4). Centrifugation was carried out, and the collected precipitate was repeatedly washed with PBS (pH 7.4) until no chromatographic peak was detected in the washing solution compared to the control group. Next, the erythrocyte membrane combined with BHD active ingredient was incubated with PBS ( $\mathrm{pH} 4.0)$ to dissociate the active ingredient from the membrane targets. After enrichment of the dissociation solution by SPE, the components were analysed by UPLC-QTOF-MS/MS to identify the compounds bound to the erythrocyte membrane.

\section{SPE}

Before MS analysis, we used SPE columns to concentrate the dissociated constituents interacting with RBCs. Inorganic salts could also be removed to protect the instrument. SPE with different types of fillers differing in retention capacities affects the recovery of various components, and thus it is necessary to optimize the SPE method. In this study, three types of SPE columns, Strata-X (polymer adsorbent), C18-N (silica sorbent), and X-HLB (hydrophilic lipophilic balance filler) and two elution procedures were carried out. In method A, the column was activated with methanol and ultra-pure water, then washed with $5 \%$ methanol (washing solution), followed by loading the dissociation solution of BHD after incubating with RBCs. After $30 \mathrm{~s}$, the samples were eluted with pure methanol and methanol containing $2 \%$ formic acid and the eluant was collected; in method B, distilled water was used as washing solution and the eluate consisted of ultrapure water containing $30 \%$ acetonitrile. The purification method was chosen based on the recovery as indicated by the HPLC peak for each sample. The recovery rate of each sample was calculated using the following formula: recovery $(\%)=$ total area of common peak after enrichment/ total area of common peak before enrichment $\times 100 \%$. A common peak indicates co-owned chromatographic peaks between BHD samples and the solution after using SPE columns.

\section{Erythrocyte activity assay}

Sprague-Dawley rats (male, 250-280 g) were provided by the Experimental Animal Center of Guangzhou University of Chinese Medicine (Guangzhou, China). The experimental protocol was conducted following the National Institute of Health Guide for the Care and Use of Laboratory Animals (NIH Publications No. 80-23, revised in 1996) and approved by Ethics Review Committee of Guangzhou University of Chinese Medicine (Guangzhou, China). After the rats were anesthetized, blood was collected into heparin tubes to prevent coagulation. The blood was centrifuged at $3200 \mathrm{rpm}$ for 10 minutes to obtain RBCs, followed by three washes with physiological saline. Calycosin, calycosin-7- $O-\beta$-D-glucoside, paeoniflorin, and 6-hydroxy kaempferol-3, 6-di-O-glucoside were dissolved to obtain gradient concentration solutions, which were incubated with RBCs at $37{ }^{\circ} \mathrm{C}$ for $1.5 \mathrm{~h}$. The control group was treated with an equal amount of physiological saline. RBCs were collected after centrifugation, and ultrapure water was added to lyse the cells. The activity of the erythrocyte membrane was tested according to the instructions of the $\mathrm{Na}^{+}-\mathrm{K}^{+}$-ATPase kit (CoWin Biosciences, China), sialic acid test kit (CoWin Biosciences, China), total superoxide dismutase (SOD) test kit (CoWin Biosciences, China) and total cholesterol kit (CoWin Biosciences, China). Each set of experiments was repeated three times.

\section{Statistical analysis}

UPLC-QTOF-MS/MS results were analysed with PeakView software (AB SCIEX). The results were presented as the mean \pm standard deviation (SD) and analysed by Student's $t$-test and one-way analysis of variance using SPSS 20.0 software (SPSS, Inc., Chicago, IL, USA). A $P$ value less than 0.05 was considered statistically significant.

\section{Results and discussion}

\section{Sample preparation}

Because many polysaccharides were found in Radix Astragali of BHD, samples prepared by the water extraction and alcohol precipitation method were highly viscous, making it difficult to perform cell membrane binding experiments. However, it was reported that alkaloids and glycosides were effective fractions of BHD. ${ }^{25}$ Therefore, after purifying the sample by water extraction and alcohol precipitation, the polysaccharide was removed by macroporous resin, and effective components such as glycosides were collected.

\section{Preparation of RBCs}

Erythrocyte membranes in healthy human and Sprague-Dawley rats are commonly used in cell membrane binding experiments. ${ }^{\text {26-28 }}$ However, limitations in obtaining human blood increases the difficulty of the experiment. Additionally, although rats are commonly used, they have a low blood volume and thus the amount of erythrocyte membranes obtained is low. Pigs are widely used to investigate vascular diseases and drug mechanisms because of their physiological similarity with humans and large blood volume. ${ }^{29}$ Therefore, porcine RBCs are a good alternative to erythrocyte membrane binding 
experiments. In our study, a suspension of porcine RBCs was obtained, and the membrane protein content was determined with a BCA kit. Only when RBC suspensions with a protein content above $0.3 \mathrm{mg} \mathrm{mL} \mathrm{m}^{-1}$ were used for subsequent experiments.

\section{SPE}

SPE is conducted to concentrate target analytes for sample simplification and transfer the dissolution medium. ${ }^{21}$ The dissociation eluent used in this study was PBS ( $\mathrm{pH} 4.0$ ), which may cause ion contamination in MS. Additionally, the volume of dissociation liquid was large but had a low content of effective components; therefore, SPE was necessary to enrich the BHD solution dissociated from RBCs. We investigated the enrichment ability of three types of SPE columns. The Strata-X SPE column is filled with a polymer adsorbent that mainly retains polar compounds. ${ }^{30} \mathrm{C} 18-\mathrm{N}$ SPE, filled with octadecyl silica, can retain both polar and non-polar compounds, indicating its high versatility. ${ }^{31,32}$ The X-HLB SPE column consists of hydrophilic lipophilic balance packing, making it suitable for isolating complex non-polar substrates to medium polarity substrates.

Our results showed that when the Strata-X and X-HLB SPE columns were combined with method A, more chromatographic peaks were obtained than by method B. However, for the C18-N SPE column, the eluate obtained by method B, with 13 common peaks showing recovery rates higher than $30 \%$, contained more ingredients than that obtained by method $\mathrm{A}$ (Fig. 1). In general, Strata-X SPE columns show poor retention of ingredients. The X-HLB SPE column also failed to retain all specific components with both the A and B elution methods. After C18-N SPE combined with elution method B, the chromatograms showed the most abundant peak information and better correlations with the pre-column sample, likely because impurities were more effectively removed. This indicates that the enrichment method of the C18-N SPE column + elution method B was the most suitable for enriching the BHD dissociation solution. Based on these results, the C18-N SPE column eluted by method B was used for enrichment experiments.

\section{Erythrocyte membrane binding and decomposition assay}

After the RBCs were incubated with BHD samples, the samples were washed, and the wash solution, including that from the control group, was collected. After SPE, the components were analysed by UPLC-QTOF-MS/MS.

The volume of BHD determines the concentration of the incubation system, which affects cell viability. To validate the concentration of BHD used in vitro, $\mathrm{Na}^{+}-\mathrm{K}^{+}$-ATPase and SOD on erythrocyte membranes were selected as pharmacodynamic indicators. Fig. S1A and $\mathrm{B} \uparrow$ revealed that $4 \mathrm{~mL}$ and $5 \mathrm{~mL}$ BHD enhanced cell activity to the greatest extent. Subsequently, optimal concentration of BHD was further analysed by MS. Compared to the mass spectrum of the BHD samples prepared in section "Sample preparation", less compound information (a total of 28 peaks) was obtained from the $4 \mathrm{~mL}$ administration group than from the $5 \mathrm{~mL}$ administration group (a total of 42 peaks), as shown in Fig. S1C and D. $\dagger$ Thus, in subsequent experiments, erythrocyte membrane binding experiments were performed using $5 \mathrm{~mL}$ of BHD samples combined with $5 \mathrm{~mL}$ of the erythrocyte membrane suspension. Furthermore, no corresponding components in BHD were found in the third to fifth sample washing solutions (Fig. S1E $\dagger$ ), resulting in no difference compared to the eluent (blank control, Fig. S1F $\dagger$ ). Thus, the RBCs can be washed three times after binding to the BHD samples. However, to ensure that the non-specific ingredients were completely removed, we washed the RBC membranes four times.

\section{Component identification and ascription}

MS is a common technique used to identify molecular structures, and the samples were analysed by MS without additional treatment after SPE. The post-enriched dissociation solution was analysed with PeakView software. By comparison of fragment ion peaks of the MS spectra with those reported by the literatures, ${ }^{19,33,34}$ four compounds were identified in the dissociation solution. As shown in Table 1 and Fig. 2, the samples scanned in positive ion mode showed information of 4 components. These compounds in BHD combined with the erythrocyte membrane were further verified by comparison with the MS spectra of standards of calycosin, paeoniflorin, 6hydroxy behenyl-3,6-di-O-glucoside, and calycosin-7-O- $\beta$-Dglucoside.

Comparison of the MS spectrograms from the dissociation of single-flavored herbs with the BHD sample revealed that the calycosin was derived from Radix Astragali. Paeoniflorin was from Radix Paeoniae Alba. Flos Carthami and Peach kernel contributed to 6-hydroxy behenol-3,6-di- $O$-glucoside and calycosin-7- $O-\beta$-D-glucoside, respectively. Interestingly, no component was found in the test solution of Angelica sinensis, Rhizome of Chuanxiong and pberetima, suggesting that these three herbs may not contribute to reduce erythrocyte aggregation.

\section{Effect of specific compounds on erythrocyte activity}

To further validate the activity of the components isolated from BHD, $\mathrm{Na}^{+}-\mathrm{K}^{+}$-ATPase, SA, SOD and $\mathrm{CHO}$ on erythrocyte membranes were selected as pharmacodynamic indicators. Because only small amounts of RBCs were needed, experiments were performed in rats. The results (Fig. 3 and Table S1 $\dagger$ ) demonstrated that the viability of $\mathrm{Na}^{+}-\mathrm{K}^{+}$-ATPase was significantly enhanced by calycosin $\left(1.0 \mu \mathrm{g} \mathrm{mL}^{-1}\right)$, paeoniflorin $(0.5 \mu \mathrm{g}$ $\left.\mathrm{mL}^{-1}\right)$, and calycosin-7-O- $\beta$-D-glucoside $\left(1.0 \mu \mathrm{g} \mathrm{mL}^{-1}\right)$, while 6hydroxy kaempferol-3,6-di-O-glucoside showed minimal effects. These four compounds significantly up-regulated the level of SA ranging from 0.1 to $10.0 \mu \mathrm{g} \mathrm{mL} \mathrm{m}^{-1}$, except for calycosin-7-O- $\beta$-Dglucoside down-regulating its level at $10.0 \mu \mathrm{g} \mathrm{mL}^{-1}$. Calycosin, calycosin-7-O- $\beta$-D-glucoside and 6-hydroxy kaempferol-3,6-di-Oglucoside could markedly higher the levels of SA and CHO in the rage of $0.1-10.0 \mu \mathrm{g} \mathrm{mL} \mathrm{m}^{-1}$, while paeoniflorin showed positive effects on SA at $1.0 \mu \mathrm{g} \mathrm{mL} \mathrm{m}^{-1}$ and $\mathrm{CHO}$ at $10.0 \mu \mathrm{g} \mathrm{mL} \mathrm{m}^{-1}$.

Fig. 3 demonstrated that as the dose increased, the response effect increased first, and then decreased, but the concentration that showed maximal effects was different. Because the sensitivity of the same indicator to different compounds is different, 

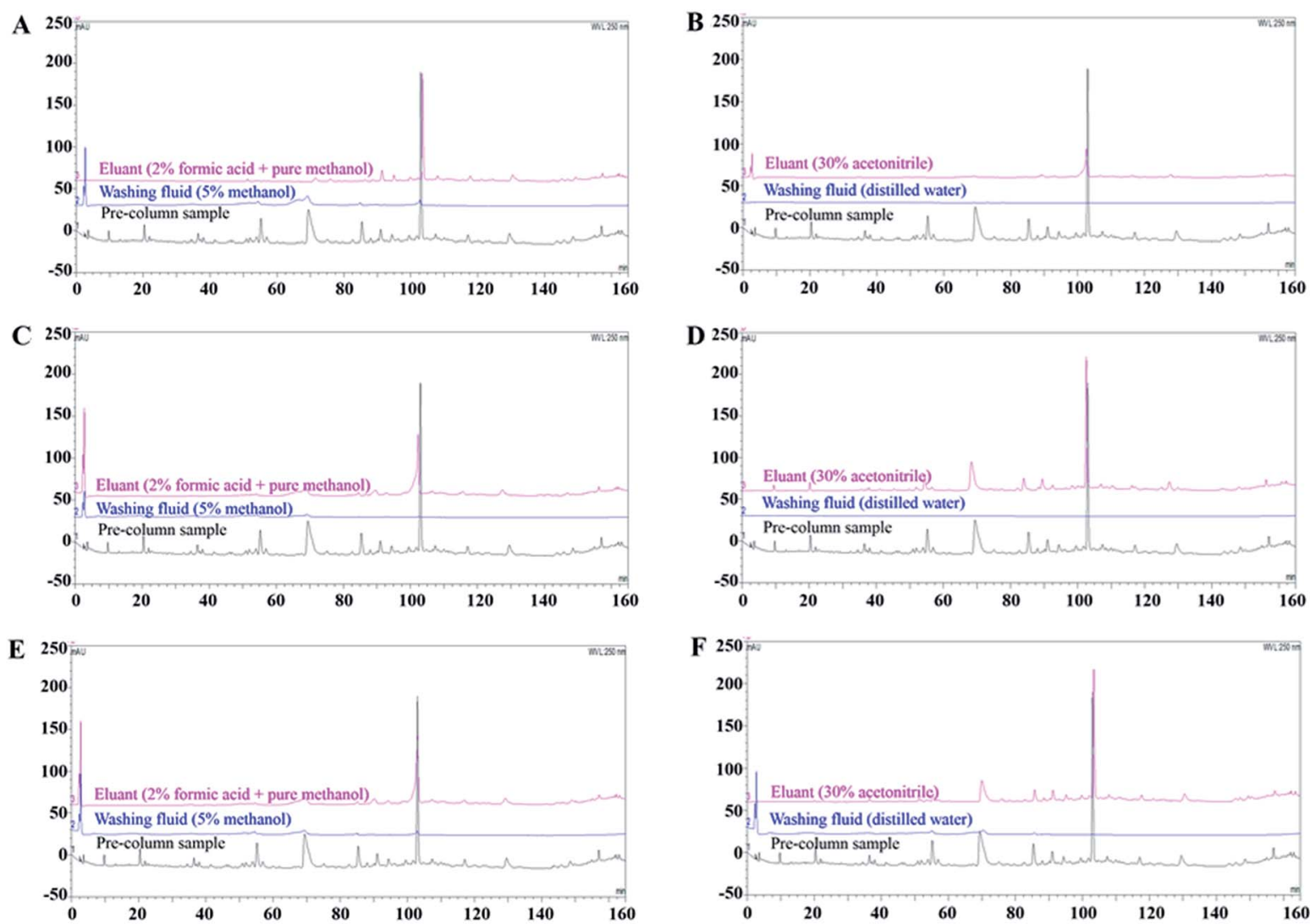

Fig. 1 Chromatograms of BHD samples processed with three types of SPE columns and two elution methods. (A) Strata-X SPE column + method A. (B) Strata-X SPE column + method B. (C) C18-N SPE column + method A. (D) C18-N SPE column + method B. (E) X-HLB SPE column + method A. (F) X-HLB SPE column + method B.

the effects of some compounds could achieve maximum at a low concentration $\left(0.1 \mu \mathrm{g} \mathrm{mL} \mathrm{m}^{-1}\right)$, such as the effect of paeoniflorin on SA and calycosin-7-O- $\beta$-D-glucoside on $\mathrm{CHO}$, but paeoniflorin cannot reduce the content of $\mathrm{CHO}$ until it reaches $10 \mu \mathrm{g} \mathrm{mL}^{-1}$. In general, the identified compounds showed maximal effect at the concentration of 0.5 or $1.0 \mu \mathrm{g} \mathrm{mL} \mathrm{m}^{-1}$. Further study on the mechanism of these compounds to reduce erythrocyte lesions requires further optimization of drug concentration.
Ischemic stroke is closely related to high RBC aggregation. ${ }^{35,36}$ Because SA is the main source of negative charges on the cell membrane surface, its reducing content decreases the negative charge on the cell surface, resulting in decreased mutual repulsion. ${ }^{37}$ Decreasing $\mathrm{Na}^{+}-\mathrm{K}^{+}$-ATPase activity on erythrocyte membranes or increasing $\mathrm{CHO}$ levels also leads to a decrease in RBC deformability, preventing microcirculation and thus increasing blood viscosity. Moreover, SOD has

Table 1 Fragment ions of combined ingredients in BHD

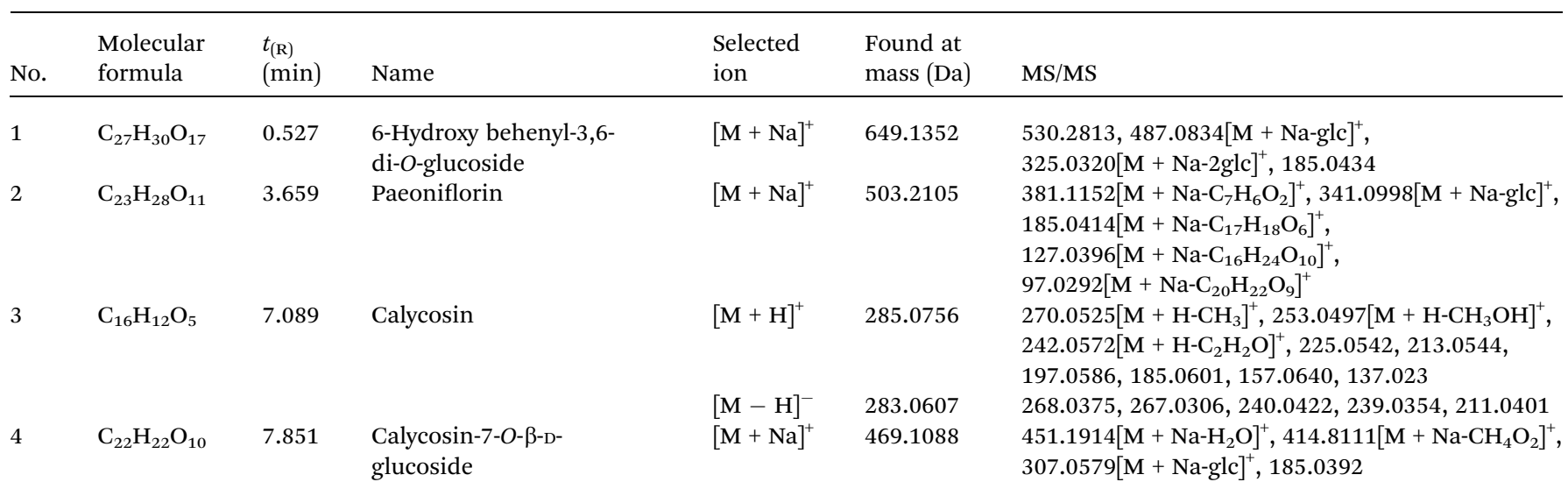



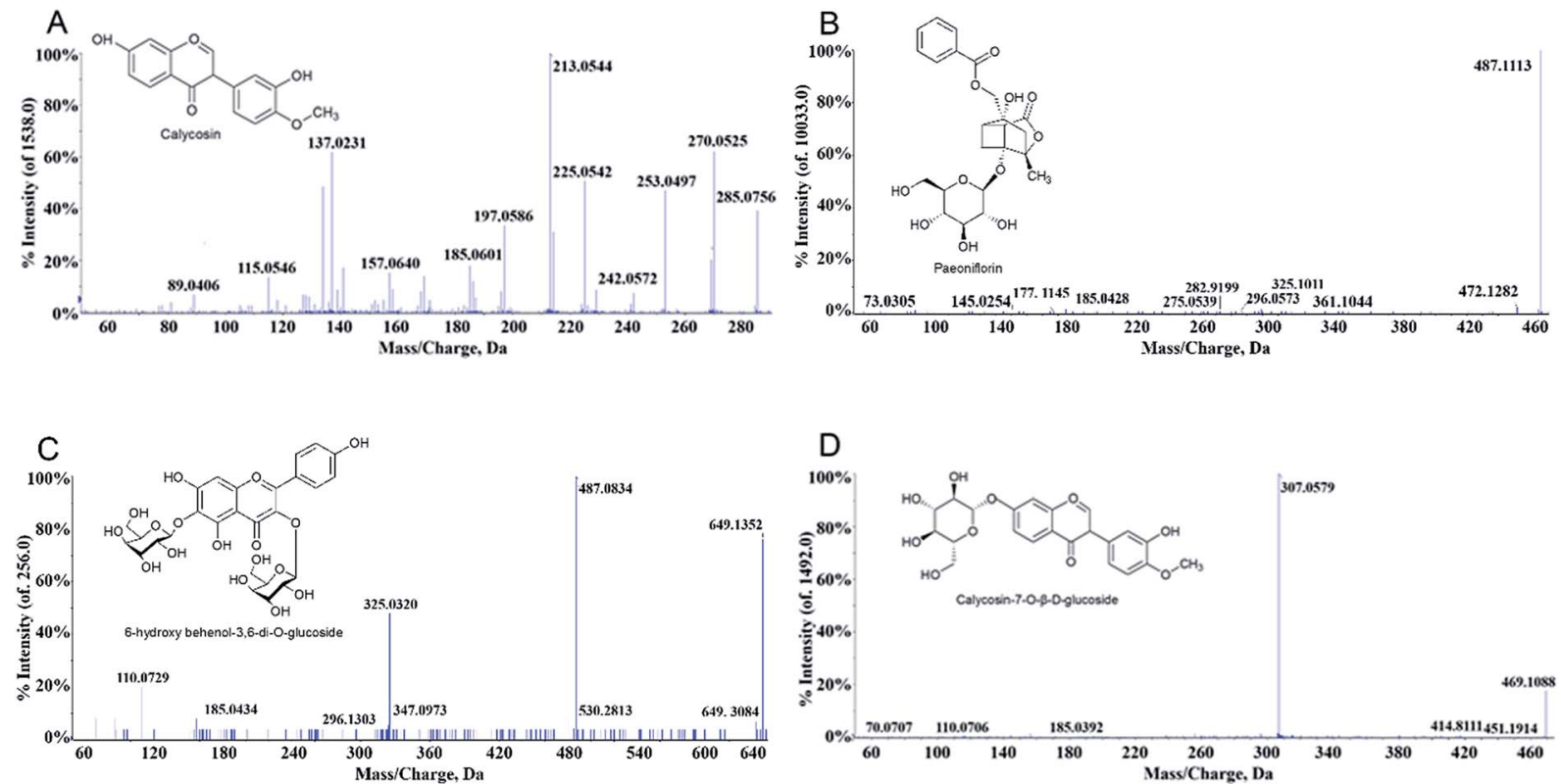

Fig. 2 MS spectra and chemical structure of the 4 compounds binding with erythrocyte membrane. (A) Calycosin. (B) Paeoniflorin. (C) 6Hydroxy behenol-3,6-di-O-glucoside. (D) Calycosin-7-O- $\beta$-D-glucoside.

a significant protective effect on RBCs, which maintains normal levels of membrane lipid fluidity. ${ }^{38}$ Taking these all together, abnormal levels of $\mathrm{Na}^{+}-\mathrm{K}^{+}$-ATPase, SA, SOD, and CHO on the erythrocyte membrane lead to increasing blood viscosity, thereby promoting the development of ischemic stroke and other diseases. Calycosin, calycosin-7-O- $\beta$-D-glucoside and
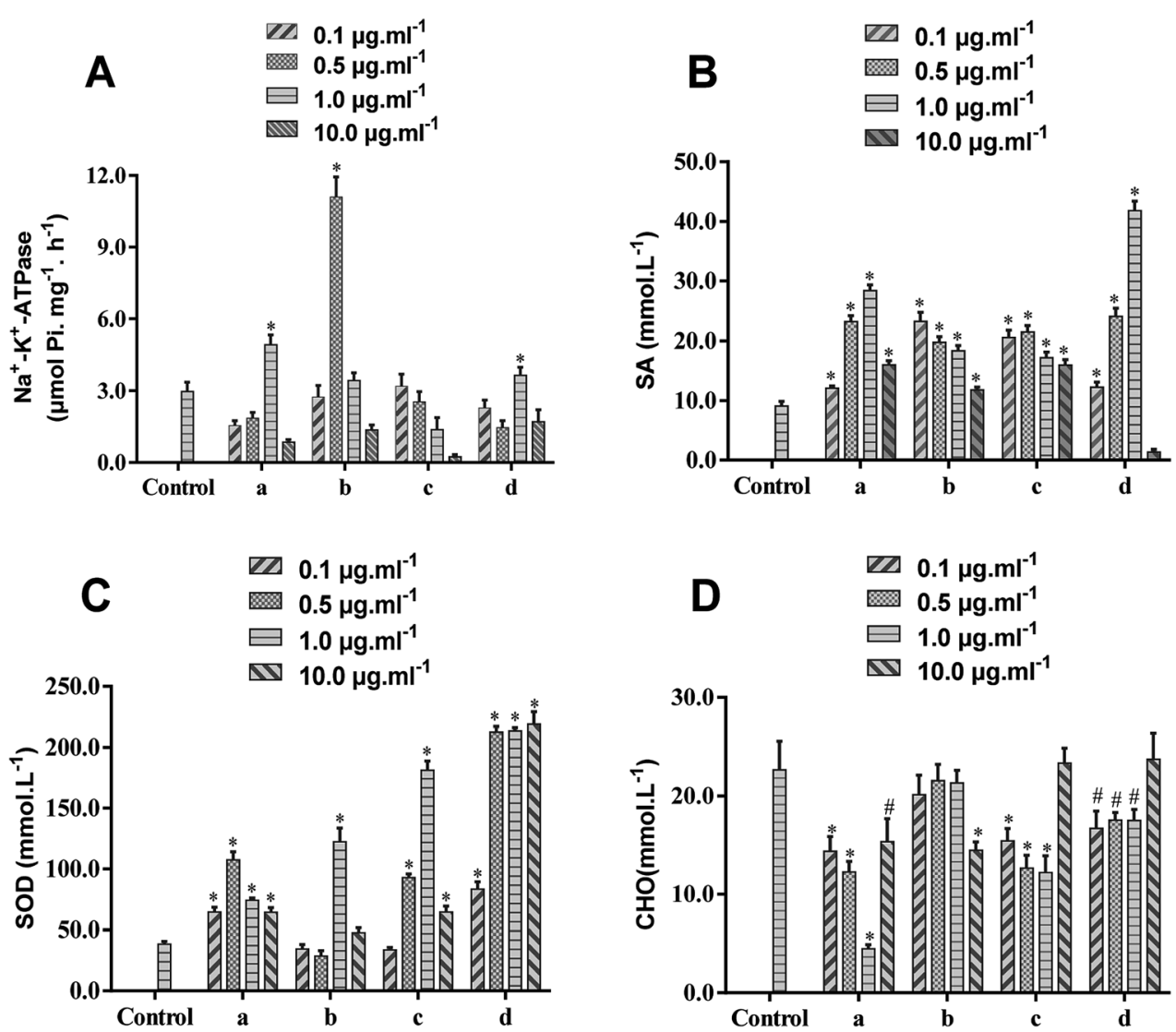

Fig. 3 Effects of the four identified compounds on (A) Na+- $\mathrm{K}^{+}$-ATPase, (B) SA, (C) SOD and (D) CHO. (a-d) Treated with calycosin, paeoniflorin, 6 -hydroxy behenol-3,6-di-O-glucoside and calycosin-7-O- $\beta$-D-glucoside, respectively. $* P<0.01$ compared with the control group, ${ }^{\#} P<0.05$ compared with the control group. 
paeoniflorin have positive effects on attenuating hypoxiareoxygenation injury of cells and protecting neurological function from ischemic stroke. ${ }^{39,40}$ Because these compounds can bind the erythrocyte membrane, we predicted that they could relieve ischemic stroke by maintaining the fluidity and deformability of RBCs, improving vascular microcirculation, reducing blood viscosity, and resisting oxidative damage. We also demonstrated the feasibility of erythrocyte membrane SPE.

\section{Conclusions}

In this study, we developed a method combining the erythrocyte membrane binding assay with SPE and UPLC-QTOF-MS/MS to screen for potential bioactive compounds that reduce the blood consistency in BHD. Calycosin, paeoniflorin, 6-hydroxy behenol-3,6-di-O-glucoside and calycosin-7-O- $\beta$-D-glucoside were found to bind the RBC membranes. The four compounds can increase the levels of $\mathrm{Na}^{+}-\mathrm{K}^{+}$-ATPase, SA and SOD and reduce the level of $\mathrm{CHO}$, suggesting that these $\mathrm{BHD}$ ingredients protect the activities of RBCs. Taking these results together, RBC affinity chromatography can be used to screen active compounds that reduce RBC lesion in herbal medicines, which would help reveal the mechanisms of many Chinese medicines for treating diseases.

However, this study only established a method for identifying the specific components of BHD binding to the erythrocyte membrane in vitro, without verifying their pharmacological activities in vivo. Further studies are necessary to demonstrate that the compounds have beneficial effects on RBCs and evaluate their activities in organisms. Moreover, we are going to use in vivo metabolites (such as drug-containing serum) and different cells for further experiments so that the established method can be improved.

\section{Conflicts of interest}

There are no conflicts to declare.

\section{Acknowledgements}

This research was financially supported by the National Natural Science Foundation of China (grant number 81573872), Guangdong Provincial Science and Technology projects (grant number 2013B032500015), Science Program for Overseas Scholar of Guangzhou University of Chinese Medicine (Torch Program XH20170108), and Project of Educational Commission of Guangdong Province (grant number 2017KZDXM018). We also thank Amanda Olsen for language help.

\section{References}

1 W. R. Wang, R. Lin, H. Zhang, Q. Q. Lin, L. N. Yang, K. F. Zhang and F. Ren, J. Ethnopharmacol., 2011, 137, 214220.

2 L. Wang, Y. Huang, J. Wu, G. Lv, L. Zhou and J. Jia, J. Pharm. Biomed. Anal., 2013, 86, 143-150.
3 Y. Chen, Y. Xiao, Z. Lin, X. Xiao, C. He, J. C. Bihl, B. Zhao, X. Ma and Y. Chen, J. Stroke Cerebrovasc. Dis., 2015, 24, 2313-2320.

4 N. Kapil, Y. H. Datta, N. Alakbarova, E. Bershad, M. Selim, D. S. Liebeskind, O. Bachour, G. H. R. Rao and A. A. Divani, Clin. Appl. Thromb./Hemostasis, 2017, 23, 301318.

5 I. Anjum, T. Kashif, M. M. Ahmed, W. Sohail, M. Sarwar and I. Khokhar, Cureus, 2018, 10, e2847.

6 J. M. Povroznik, J. E. Ozga, C. V. Haar and E. B. EnglerChiurazzi, Behav. Pharmacol., 2018, 29, 638-653.

7 S. S. Hashem, S. M. Helmy, N. M. El-Fayomy, M. I. Oraby, M. Menshawy, N. A. Dawood and H. S. Hashem, The Egyptian journal of neurology, psychiatry and neurosurgery, 2018, 54, 18.

8 S. Staessens, F. Denorme, O. Francois, L. Desender, T. Dewaele, P. Vanacker, H. Deckmyn, K. Vanhoorelbeke, T. Andersson and S. F. De Meyer, Haematologica, 2019, DOI: $10.3324 /$ haematol.2019.219881.

9 S. Duffy, R. McCarthy, M. Farrell, S. Thomas, P. Brennan, S. Power, A. O'Hare, L. Morris, E. Rainsford, E. MacCarthy, J. Thornton and M. Gilvarry, Stroke, 2019, 50, 1156-1163.

10 J. W. Weisel and R. I. Litvinov, J. Thromb. Haemostasis, 2019, 17, 271-282.

11 H. Liu, Y. Y. Peng, F. Y. Liang, S. Chen, P. B. Li, W. Peng, Z. Z. Liu, C. S. Xie, C. F. Long and W. W. Su, Biotechnol. Biotechnol. Equip., 2014, 28, 140-146.

12 A. P. McNamee, G. D. Tansley and M. J. Simmonds, Microvasc. Res., 2018, 120, 1-7.

13 J. Zheng, B. L. Liu, Q. X. Lun, W. J. Yao, Y. F. Zhao, W. Xiao, W. Z. Huang, Y. H. Wang, J. Li and P. F. Tu, Oxid. Med. Cell. Longevity, 2016, 11, DOI: 10.1155/2016/2603219.

14 T. Tashima, Bioorg. Med. Chem. Lett., 2018, 28, 3015-3024.

15 S. Balzan, G. D'Urso, S. Ghione, A. Martinelli and U. Montali, Life Sci., 2000, 67, 1921-1928.

16 K. Y. Lu, X. Chen, W. W. Zhu, X. N. Mao, Y. Yang, J. Qiu, M. S. Zhang and R. Cheng, Pediatr. Pulmonol., 2019, 54, 280-288.

17 I. Galvez, L. Martin-Cordero, M. D. Hinchado, A. AlvarezBarrientos and E. Ortega, Brain, Behav., Immun., 2019, 80, 564-572.

18 S. L. Li, P. Li, L. H. Sheng, R. Y. Li, L. W. Qi and L. Y. Zhang, J. Pharm. Biomed. Anal., 2006, 41, 576-581.

19 F. Liao, Y. Meng, H. Zheng, D. He, X. Shen, J. Yu, Y. Wu and L. Wang, Talanta, 2018, 490-500.

20 S. Liu, Z. Tan, P. Li, X. Gao, Y. Zeng and S. Wang, J. Pharm. Biomed. Anal., 2016, 121, 56-62.

21 Y. Bu, X. He, Q. Hu, C. Wang, X. Xie and S. Wang, Sci. Rep., 2017, 7, 3569.

22 C. Temporini, G. Massolini, G. Marucci, C. Lambertucci, M. Buccioni, R. Volpini and E. Calleri, Anal. Bioanal. Chem., 2013, 405, 837-845.

23 F. Liao, Y. Meng, H. Zheng, D. He, X. Shen, J. Yu, Y. Wu and L. Wang, Talanta, 2018, 179, 490-500.

24 N. K. Jain, Artif. Cells, Nanomed., Biotechnol., 2013, 41, 309314. 
25 J. Yang, Y. Liang, S. P. Zhang and C. Q. Deng, Chin. J. Exp. Tradit. Med. Formulae, 2006, 12, 28-30.

26 S. L. Li, P. Li, L. H. Sheng, R. Y. Li, L. W. Qi and L. Y. Zhang, J. Pharm. Biomed. Anal., 2006, 41, 576-581.

27 J. Wang, Z. G. Huang, H. Cao, Y. T. Wang, P. Hui, C. Hoo and S. P. Li, J. Sep. Sci., 2008, 31, 1173-1180.

28 Y. Chen, N. Zhang, J. Ma, Y. Zhu, M. Wang, X. Wang and P. Zhang, J. Pharm. Biomed. Anal., 2016, 117, 178-183.

29 M. J. Zurbano, B. Fuste, G. Arderiu, G. Escolar, A. Ordinas and M. Diaz-Ricart, J. Thromb. Haemostasis, 2003, 1, 24112418.

30 L. Mut, T. Grobosch, T. Binscheck-Domass and W. Frenzel, Biomed. Chromatogr., 2015, 29, 935-952.

31 J. Li, X. Zheng, H. Wang and H. Qiu, Chin. J. Chromatogr., 2017, 35, 1211-1215.

32 Y. Hao, X. Gao and W. Xia, Molecules, Basel, Switzerland, 2018, vol. 23.
33 L. E-Hu, Q. Lian-Wen, C. Xiao-Lan, P. Yong-Bo and L. Ping, Biomed. Chromatogr., 2010, 24, 125-131.

34 L. E-Hu, Q. Lian-Wen, P. Yong-Bo, C. Xiao-Lan, W. Qian, L. Ping and L. Chang-Yin, Biomed. Chromatogr., 2010, 23, 828-842.

35 M. Fisher and H. J. Meiselman, Stroke, 1991, 22, 1164-1169.

36 N. Tanahashi, F. Gotoh, M. Tomita, T. Shinohara, Y. Terayama, B. Mihara, K. Ohta and M. Nara, Stroke, 1989, 20, 1202-1207.

37 A. Patel, S. Tiwari and P. K. Jha, J. Biomol. Struct. Dyn., 2018, 1-10, DOI: 10.1080/07391102.2018.1461690.

38 E. Pytel, M. Olszewska-Banaszczyk, M. Koter-Michalak and M. Broncel, Biochim. Biol. Cell., 2013, 91, 315-318.

39 W. W. Zhang, F. Xu, D. Wang, J. Ye and S. Q. Cai, Chin. J. Nat. Med., 2018, 16, 194-202.

40 S. Fu, Y. Gu, J. Q. Jiang, X. Chen, M. Xu, X. Chen and J. Shen, J. Ethnopharmacol., 2014, 155, 692-701. 